\title{
A FLUX-CORRECTED FINITE ELEMENT METHOD FOR CHEMOTAXIS PROBLEMS
}

\author{
R. STREHL ${ }^{1}$, A. SOKOLOV ${ }^{1}$, D. KUZMIN ${ }^{1}$, AND S. TUREK $^{1}$
}

\begin{abstract}
An implicit flux-corrected transport (FCT) algorithm has been developed for a class of chemotaxis models. The coefficients of the Galerkin finite element discretization has been adjusted in such a way as to guarantee mass conservation and keep the cell density nonnegative. The numerical behaviour of the proposed highresolution scheme is tested on the blow-up problem for a minimal chemotaxis model with singularities. It has also been shown that the results for an Escherichia coli chemotaxis model are in good agreement with the experimental data reported in the literature.
\end{abstract}

2000 Mathematics Subject Classification: 35B36; 65N30; 92C15; 92C17.

Keywords: chemotaxis models, pattern formation, flux limiters, finite elements.

\section{Introduction}

Chemotaxis, an oriented movement towards or away from regions of higher concentrations of certain chemicals, plays a vitally important role in the evolution of many living organisms. The chemotactical response gives numerous creatures, ranging from bacteria and protozoa to tissue cells, a chance to find more favourable locations in their environments. This feature improves their ability to search for food, detect the location of mates or escape danger. Chemotaxis finds many medical and biological applications, including bacteria/cells aggregation and pattern formation processes, tumour growth, etc.

The first mathematical description of chemotactical processes was given by Keller and Segel $[14,15]$, who modeled the aggregation of the slime mold amoeba Dictyostelium discoideum. Their work was followed by the development of sophisticated models for various chemotaxis problems $[2,5,13,20,27]$. The numerical treatment of chemotaxis equations has also been addressed by many authors $[7,9,10,16,23,28]$. However, some implementation aspects still call for further research. In particular, it is difficult to design a robust, accurate, and efficient numerical algorithm that does not produce negative densities or concentrations [7]. In the present paper, positivity constraints for the Galerkin finite element discretization are enforced using a generalized flux-corrected transport (FCT) algorithm [4, 17, 19, 29].

A representative class of chemotaxis models based on advection-reaction-diffusion equations is considered in what follows. Following the notation of [13], the nonlinear PDE systems to be solved in a two-dimensional domain $\Omega \subset \mathbb{R}^{2}$ are written in the unified form

$$
\begin{aligned}
& u_{t}=\nabla \cdot(D(u) \nabla u-A(u) B(c) C(\nabla c))+q(u) \text { in } \Omega, \\
& c_{t}=d \Delta c-s(u) c+g(u) u \text { in } \Omega,
\end{aligned}
$$

\footnotetext{
${ }^{1}$ Institute of Applied Mathematics, LS III, TU Dortmund, Vogelpothsweg 87, D-44227 Dortmund, Germany. E-mail: robert.strehl@math.uni-dortmund.de, asokolow@math.uni-dortmund.de
} 
where $u(\boldsymbol{x}, t)$ denotes the cell density and $c(\boldsymbol{x}, t)$ is the chemoattractant concentration. The functional dependence of the involved coefficients on $u$ and $c$ defines a particular model. A variety of complex chemotactical processes can be modelled in this way $[2,5,16,20,27]$.

The above transport equation for $u$ and the reaction-diffusion equation for $c$ are endowed with the initial conditions

$$
\left.u\right|_{t=0}=u_{0},\left.\quad c\right|_{t=0}=c_{0} \quad \text { in } \quad \Omega,
$$

and homogeneous Neumann boundary conditions are prescribed on the boundary $\Gamma$ of $\Omega$

$$
\boldsymbol{n} \cdot(D(u) \nabla u-A(u) B(c) C(\nabla c))=\boldsymbol{n} \cdot \nabla c=0 \quad \text { on } \quad \Gamma .
$$

One of the numerical problems to be dealt with is due to the rapid growth of solutions to system (1.1)-(1.2) in a small neighbourhood of certain points or curves. In particular, the blow-up phenomenon, or a singular spiky behaviour of exact solutions, may give rise to nonphysical oscillations if the employed numerical scheme is not guaranteed to satisfy the discrete maximum principle (DMP). The available numerical techniques include various positivity-preserving finite volume and finite element schemes [7, 11, 25], operator-splitting, fractional step algorithms $[23,28]$, interior penalty discontinuous Galerkin methods [9, 10], and cell-overcrowding prevention models $[6,8,22]$. The flux-corrected transport paradigm described in Section 2 represents a promising new approach to the blow-up problem.

Another interesting application of the proposed methodology is the numerical prediction of bacteria pattern formations. The nonlinear dependence of $B(c)$ on the chemoattractant concentration $c$ can produce travelling waves [3, 24]. Attracting and repulsing substances behave in different ways. As shown by the numerical study of Aida et al. [1,2] and confirmed experimentally, the pattern for small values of the parameter $\chi=B(c)=$ const resembles a honeycomb, stripe or perforated stripe, while a chaotic spot pattern is observed for large values of $\chi$. In Section 3, the proposed FEM-FCT algorithm is applied to 2D pattern formation problems. The results presented are in good agreement with the available experimental data.

\section{Flux-corrected transport}

A segregated approach to the numerical solution of the nonlinear model problem (1.1)-(1.2) was adopted. In each time step, the transport equation for the chemoattractant concentration $c(\boldsymbol{x}, t)$ is solved prior to that for the cell density $u(\boldsymbol{x}, t)$. Both equations are written in weak form and discretized in space using (conforming) bilinear finite elements. The discretization in time is performed by the implicit Euler method; Crank-Nicolson and fractional step schemes will be considered in a forthcoming paper. The system of linearized algebraic equations consists of two decoupled subproblems for the unknowns $u^{n+1}$ and $c^{n+1}$ at time $t_{n+1}$ :

$$
\begin{aligned}
{\left[\boldsymbol{M}(1)+\Delta t \boldsymbol{L}\left(D^{n}\right)-\Delta t \boldsymbol{K}\left(c^{n}\right)\right] u^{n+1} } & =\boldsymbol{M}(1) u^{n}+\Delta t \boldsymbol{q}^{n}, \\
{\left[\boldsymbol{M}(1)+\Delta t \boldsymbol{L}(d)-\Delta t \boldsymbol{M}\left(s^{n}\right)\right] c^{n+1} } & =\boldsymbol{M}(1) c^{n}+\Delta t \boldsymbol{M}\left(g^{n}\right) u^{n},
\end{aligned}
$$

where $\boldsymbol{M}(\cdot)$ denotes the (consistent) mass matrix, $\boldsymbol{L}(\cdot)$ is a discrete diffusion operator, and $\boldsymbol{K}(c)$ is a discrete transport operator due to the chemotactical flux $A(u) B(c) C(\nabla c)$. The 
entries of $\boldsymbol{M}(\cdot), \boldsymbol{L}(\cdot), \boldsymbol{K}(c)$ and $\boldsymbol{q}^{n}$ are defined in (2.3)-(2.6). In (2.1)-(2.2) the setting $D^{n}=D\left(u^{n}\right), s^{n}=s\left(u^{n}\right)$ and $g^{n}=g\left(u^{n}\right)$ is used.

Given a set of piecewise-polynomial basis functions $\left\{\varphi_{i}\right\}$, the standard Galerkin discretization yields the following formulae for the coefficients of the matrices $\boldsymbol{M}, \boldsymbol{L}, \boldsymbol{K}$ and the vector $\boldsymbol{q}^{n}$ :

$$
\begin{aligned}
m_{i j}(\psi) & =\int_{\Omega} \varphi_{i} \varphi_{j} \psi \mathrm{d} \mathbf{x}, \quad \psi \in\{1, s(u), g(u)\}, \\
l_{i j}(\psi) & =\int_{\Omega} \nabla \varphi_{i} \cdot \nabla \varphi_{j} \psi \mathrm{d} \mathbf{x}, \quad \psi \in\{D(u), d\}, \\
k_{i j}(c) & =\int_{\Omega} \nabla \varphi_{i} \cdot A\left(\varphi_{j}\right) B(c) C(\nabla c) \mathrm{d} \mathbf{x}, \\
q_{i}^{n} & =\int_{\Omega} \varphi_{i} q_{j}\left(u^{n}\right) \mathrm{d} \mathbf{x} .
\end{aligned}
$$

In formula (2.5), the discontinuous concentration gradient $\nabla c$ can be replaced by a superconvergent approximation constructed using (slope-limited) reconstruction techniques [18].

As was shown by Kuzmin et al. [18, 19, 17], positivity constraints can be readily enforced at the discrete level using a conservative manipulation of the matrices $\boldsymbol{M}$ and $\boldsymbol{K}$. The former is approximated by its diagonal counterpart $\boldsymbol{M}_{L}$ constructed using row-sum mass lumping

$$
\boldsymbol{M}_{L}:=\operatorname{diag}\left\{m_{i}\right\}, \quad m_{i}=\sum_{j} m_{i j}(1)
$$

Next, all negative off-diagonal entries of $\boldsymbol{K}$ are eliminated by adding an artificial diffusion operator $\boldsymbol{D}$. For conservation reasons, this matrix must be symmetric with zero row and column sums. For any pair of neighbouring nodes $i$ and $j$, the entry $d_{i j}$ is defined as $[18,19]$

$$
d_{i j}=\max \left\{-k_{i j}, 0,-k_{j i}\right\}, \quad j \neq i .
$$

Note that $d_{j i}=d_{i j}$, so that the operator $\boldsymbol{D}$ is a symmetric matrix. The diagonal coefficients $d_{i i}$ are defined so that the row and column sums of $\boldsymbol{D}$ are equal to zero

$$
d_{i i}=-\sum_{j \neq i} d_{i j}
$$

The result is a positivity-preserving discretization of low order. By construction, the added perturbation to the discrete problem admits a conservative decomposition into a sum of internodal fluxes. The mass lumping error and artificial diffusion received by the node $i$ satisfy

$$
\begin{aligned}
\left(M(1) u-M_{L} u\right)_{i} & =\sum_{j} m_{i j} u_{j}-m_{i} u_{i}=\sum_{j \neq i} m_{i j}\left(u_{j}-u_{i}\right) \\
(D u)_{i}=\sum_{j} d_{i j} u_{j} & =\sum_{j \neq i} d_{i j} u_{j}+d_{i i} u_{i}=\sum_{j \neq i} d_{i j}\left(u_{j}-u_{i}\right) .
\end{aligned}
$$


Let $f$ denote the difference between the residuals of the low-order scheme and that of the underlying Galerkin approximation. By virtue of the above flux decomposition, we have

$$
f_{i}=\sum_{j \neq i} f_{i j}, \quad f_{j i}=-f_{i j}, \quad \forall j \neq i
$$

To achieve a high resolution while keeping the scheme positivity-preserving, each flux is multiplied by a solution-dependent correction factor $\alpha_{i j} \in[0,1]$ and inserted into the right-hand side of the nonoscillatory low-order scheme. The original Galerkin discretization corresponds to the setting $\alpha_{i j}:=1$. It may be used in regions where the numerical solution is smooth and well-resolved. The setting $\alpha_{i j}:=0$ is appropriate in the neighborhood of steep fronts.

In essence, the off-diagonal entries of the sparse matrices $\boldsymbol{M}$ and $\boldsymbol{K}$ are replaced by

$$
m_{i j}^{*}:=\alpha_{i j} m_{i j}, \quad k_{i j}^{*}:=k_{i j}+\left(1-\alpha_{i j}\right) d_{i j},
$$

while the diagonal coefficients of the flux-corrected Galerkin operators are given by

$$
m_{i i}^{*}:=m_{i}-\sum_{j \neq i} \alpha_{i j} m_{i j}, \quad k_{i i}^{*}:=k_{i i}-\sum_{j \neq i}\left(1-\alpha_{i j}\right) d_{i j} .
$$

In implicit FEM-FCT schemes [17, 18, 19], the optimal values of $\alpha_{i j}$ are determined using Zalesak's algorithm [29]. The limiting process begins with cancelling all fluxes that are diffusive in nature and tend to flatten the solution profiles. The required modification is

$$
f_{i j}:=0 \quad \text { if } \quad f_{i j}\left(u_{j}-u_{i}\right)>0
$$

where $u$ is a positivity-preserving solution of low order $[17,18,19]$. The remaining fluxes are truly antidiffusive, and the computation of $\alpha_{i j}$ involves the following algorithmic steps:

1. Compute the sums of positive/negative antidiffusive fluxes into node $i$

$$
P_{i}^{+}=\sum_{j \neq i} \max \left\{0, f_{i j}\right\}, \quad P_{i}^{-}=\sum_{j \neq i} \min \left\{0, f_{i j}\right\}
$$

2. Compute the distance to a local extremum of the auxiliary solution $u$

$$
Q_{i}^{+}=\max \left\{0, \max _{j \neq i}\left(u_{j}-u_{i}\right)\right\}, \quad Q_{i}^{-}=\min \left\{0, \min _{j \neq i}\left(u_{j}-u_{i}\right)\right\} .
$$

3. Compute the nodal correction factors for the net increment to node $i$

$$
R_{i}^{+}=\min \left\{1, \frac{m_{i} Q_{i}^{+}}{\Delta t P_{i}^{+}}\right\}, \quad R_{i}^{-}=\min \left\{1, \frac{m_{i} Q_{i}^{-}}{\Delta t P_{i}^{-}}\right\}
$$

4. Check the sign of the antidiffusive flux and apply the correction factor

$$
\alpha_{i j}= \begin{cases}\min \left\{R_{i}^{+}, R_{j}^{-}\right\}, & \text {if } f_{i j}>0 \\ \min \left\{R_{i}^{-}, R_{j}^{+}\right\}, & \text {otherwise. }\end{cases}
$$


In the context of chemotaxis problems, the above limiting strategy ensures that the cell density $u(\boldsymbol{x}, t)$ and the concentration $c(\boldsymbol{x}, t)$ remain nonnegative. However, the resultant algebraic systems are strongly nonlinear and must be solved iteratively. As a remedy, the antidiffusive fluxes $f_{i j}$ for an implicit FCT algorithm can be linearized about a low-order predictor, as proposed by Kuzmin [17]. This linearized version of FEM-FCT is the method that we use to solve our system (1.1)-(1.2) in the present paper. In contrast to nonlinear FCT algorithms, antidiffusive flux correction is done explicitly after calculation of the loworder solution. Therefore, it is readily applicable to linear and nonlinear problems alike. For a detailed presentation of the FEM-FCT methodology, including theoretical analysis (stability, positivity, convergence) and technical implementation details (data structures, matrix assembly), we refer the interested reader to $[17,18,19]$ and other publications by Kuzmin et al.

\section{Numerical results}

In this section, the developed FEM-FCT algorithm is applied to chemotaxis models that call for the use of positivity-preserving discretization techniques.

\subsection{Blow-up in the center of the domain}

The minimal Keller-Segel chemotaxis model

$$
\begin{aligned}
& u_{t}=\Delta u-\nabla \cdot(u \nabla c), \\
& c_{t}=\Delta c-c+u,
\end{aligned}
$$

can be written in the form (1.1)-(1.2). The corresponding parameter settings are as follows:

$$
\begin{array}{cl}
A(u)=u, & B(c)=1, \quad C(\nabla c)=\nabla c, \quad D(u)=1, \\
d=1, & s(u)=1, \quad g(u)=1, \quad q(u)=0 .
\end{array}
$$

The following bell-shaped initial conditions [7] are prescribed in $\Omega=(0,1)^{2}$ at $t=0$

$$
\begin{aligned}
& u_{0}(x, y)=1000 e^{-100\left((x-0.5)^{2}+(y-0.5)^{2}\right)}, \\
& c_{0}(x, y)=500 e^{-50\left((x-0.5)^{2}+(y-0.5)^{2}\right)} .
\end{aligned}
$$

The radially symmetric solution to the initial boundary value problem (3.1)-(3.3) has a peak in the center of the domain $\Omega$, where the blow-up of $u$ and $c$ occurs in finite time [12, 26]. The numerical solutions to the blow-up problem are computed on a uniform grid of bilinear finite elements. The mesh size and time step are given by $h=1 / 128$ and $\Delta t=10^{-6}$, respectively. Snapshots of the results obtained with the standard Galerkin discretization of system (3.1)(3.2) are displayed in Fig. 3.1. The two diagrams in Fig. 3.2 show the distribution of the cell density $u$ along the horizontal line $y=0.5$ at two time instants. Note that $u$ becomes negative at a certain intermediate time. The nonphysical negative values grow rapidly as time evolves, which leads to an abnormal termination of the simulation run. 

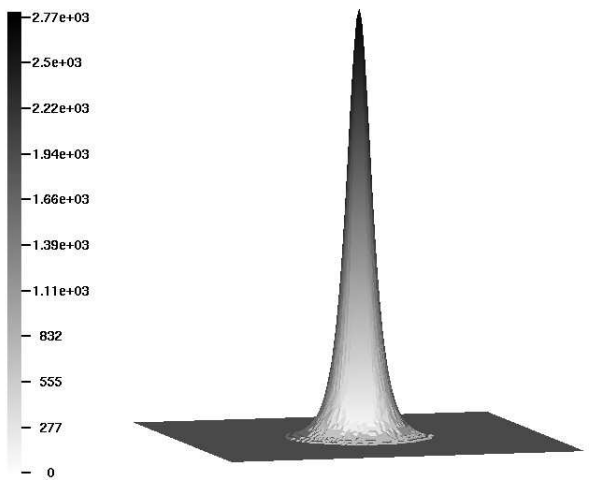

(a) $t=10^{-5}$
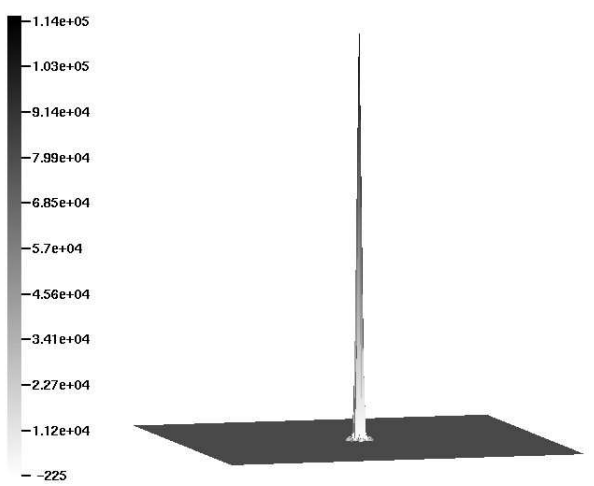

(c) $t=6 \cdot 10^{-5}$

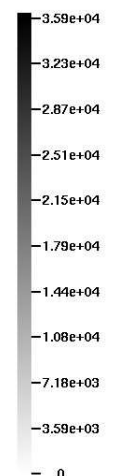

0

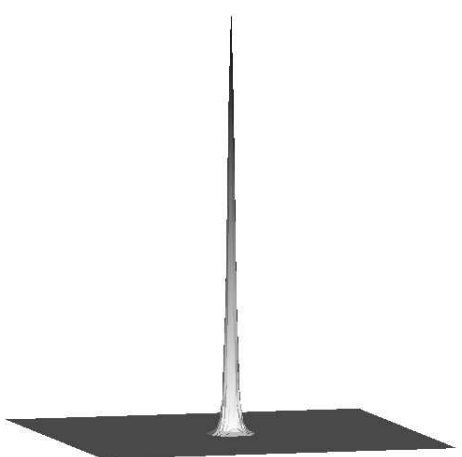

(b) $t=4 \cdot 10^{-5}$

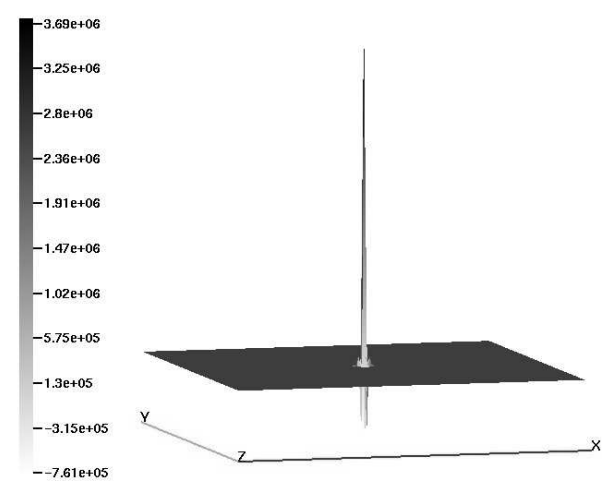

(d) $t=1.2 \cdot 10^{-4}$

Fig. 3.1. Blow-up in the center, standard Galerkin scheme, $h=\frac{1}{128}, \Delta t=10^{-6}$.

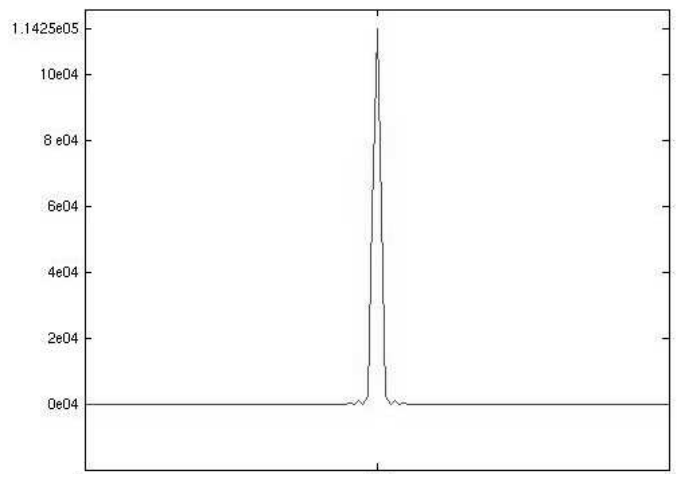

(a) $t=6 \cdot 10^{-5}$

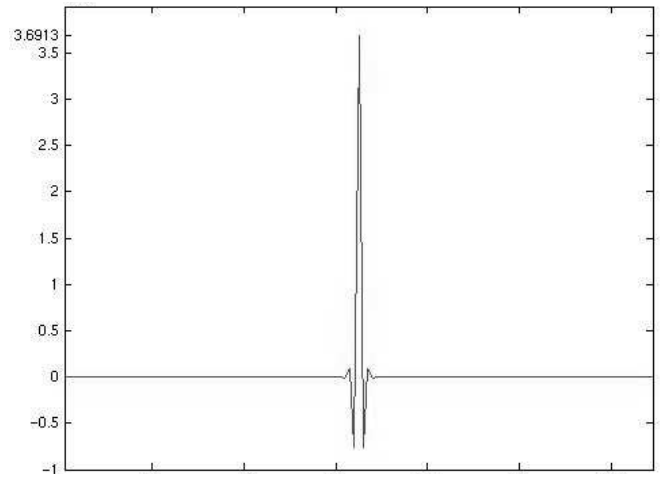

(b) $t=1.2 \cdot 10^{-4}$

Fig. 3.2. Blow-up in the center, Galerkin solution at $y=0.5, h=\frac{1}{128}, \Delta t=10^{-6}$.

Next, we apply the FCT correction to the discretized form of the minimal chemotaxis system (3.1)-(3.2) and perform simulations with the same parameter settings as before. The numerical solutions presented in Figs. 3.3 and 3.4 are seen to be positive and nonoscillatory. 


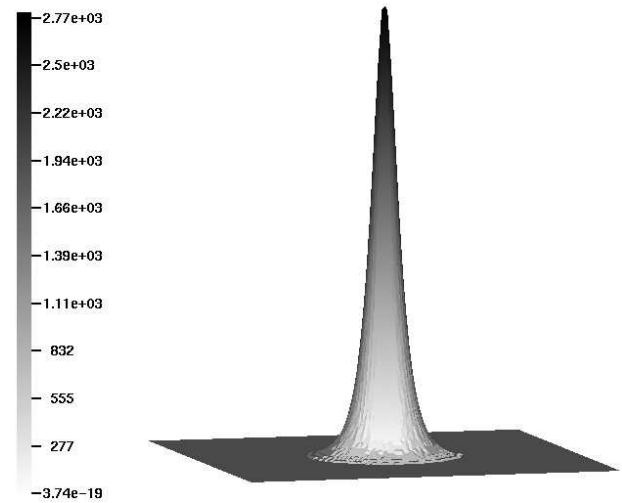

(a) $t=10^{-5}$

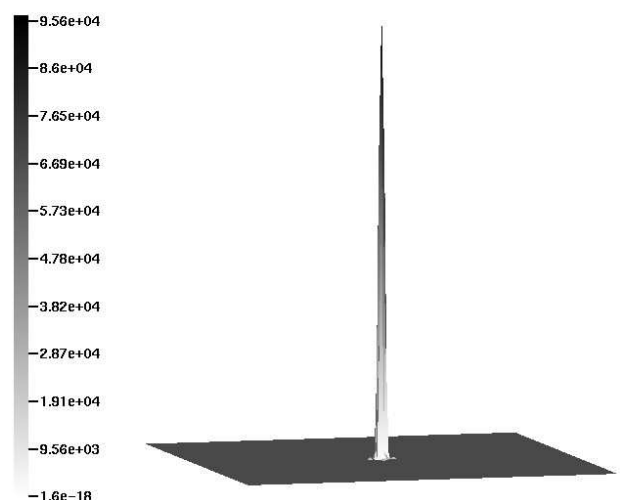

(c) $t=6 \cdot 10^{-5}$

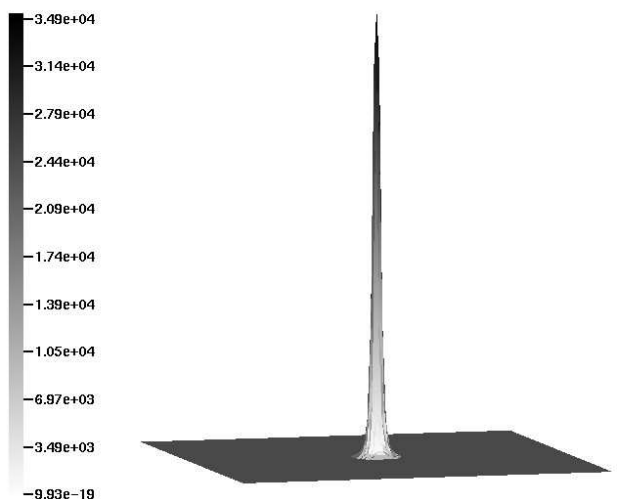

(b) $t=4 \cdot 10^{-5}$

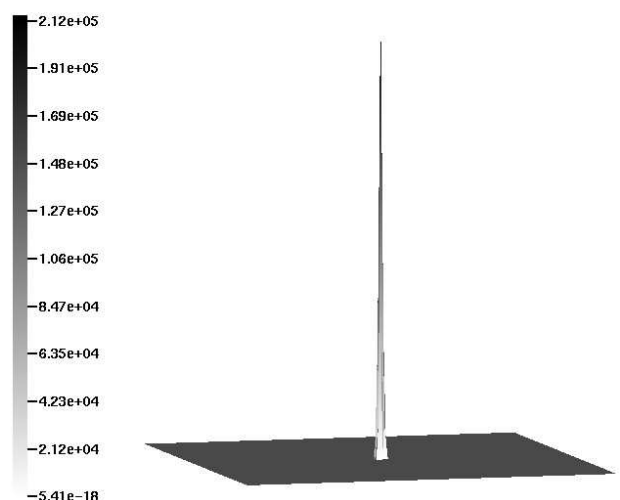

(d) $t=1.2 \cdot 10^{-4}$

Fig. 3.3. Blow-up in the center, FEM-FCT scheme, $h=\frac{1}{128}, \Delta t=10^{-6}$.

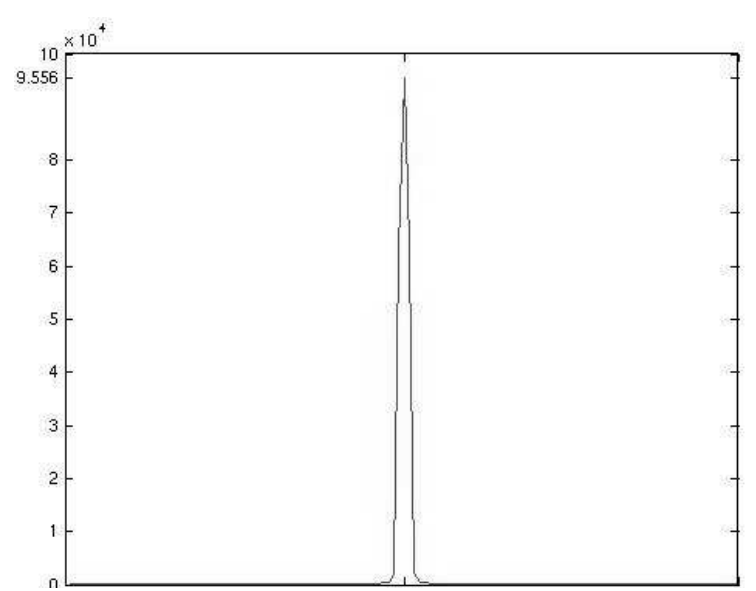

(a) $t=6 \cdot 10^{-5}$

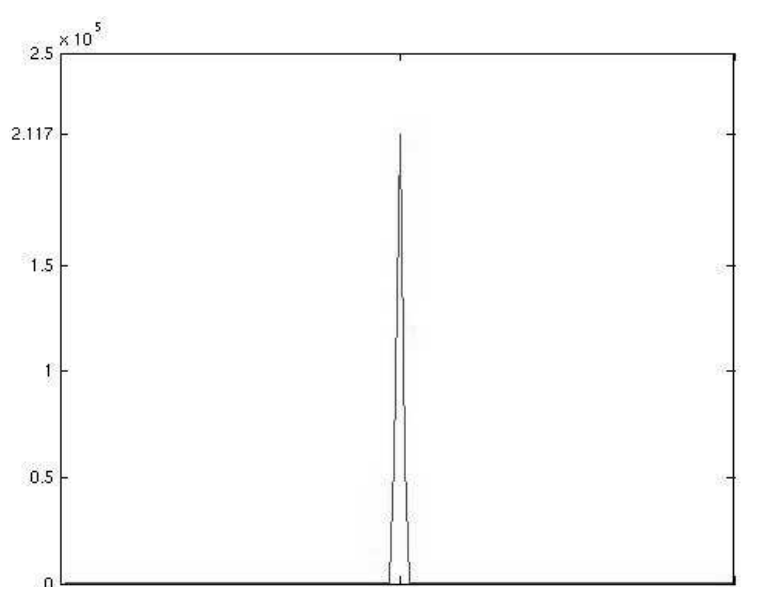

(b) $t=1.2 \cdot 10^{-4}$

Fig. 3.4. Blow-up in the center, FEM-FCT solution at $y=0.5, h=\frac{1}{128}, \Delta t=10^{-6}$.

The accuracy of a finite element approximation can be easily improved by means of local mesh refinement in underresolved regions. Since the solution of system (3.1)-(3.2) blows up in the center of the square domain, it is worthwhile to refine the mesh around this point, so as to achieve a higher resolution of the growing peak. For a fair comparison, the number 
of elements (degrees of freedom) should not exceed that for the uniform grid employed previously. The FEM-FCT solution presented in Fig. 3.5 (b) was computed on a nonuniform mesh constructed from that shown in Fig. 3.5 (a) using 5 levels of global refinement. The total number of elements is $13,312<128^{2}$. Due to the higher mesh density around the point of blow-up, the peak of the cell density is approximately twice as high as that in Fig. 3.3 (d). The peak heights variation with uniform and adaptive mesh refinement is illustrated by the diagram in Fig. 3.6.

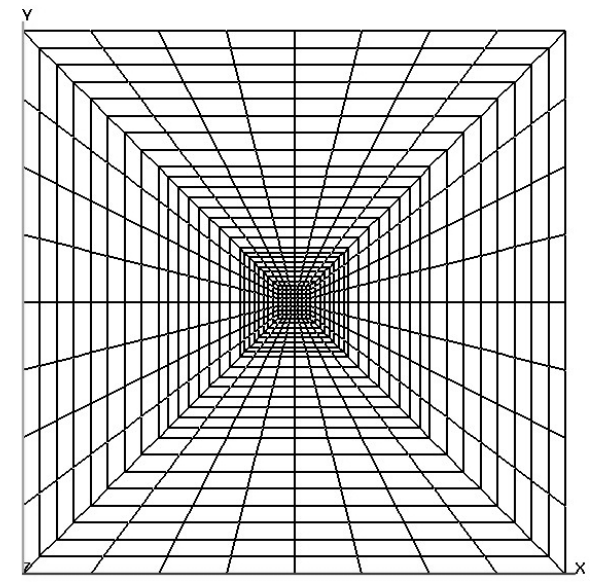

(a) adaptive mesh, level $3, h \leqslant 1 / 8$

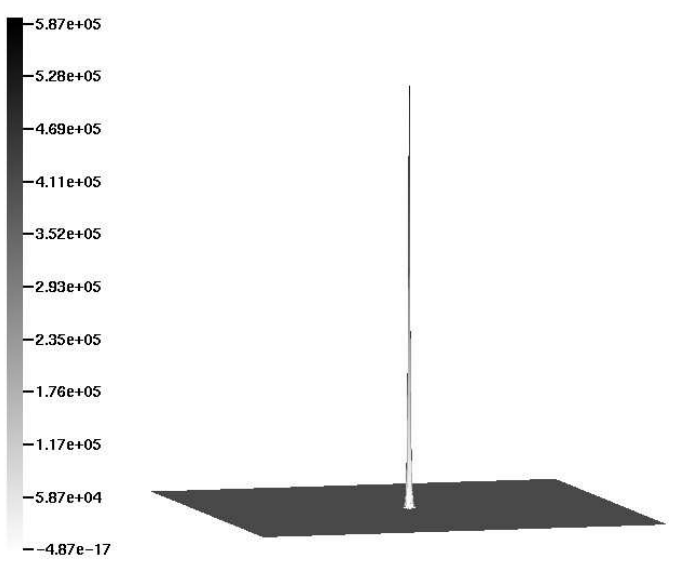

(b) cell density $u$ at $t=1.2 \cdot 10^{-4}$

Fig. 3.5. Blow-up in the center, adaptive FEM-FCT scheme, 13,312 elements, $\Delta t=10^{-6}$.

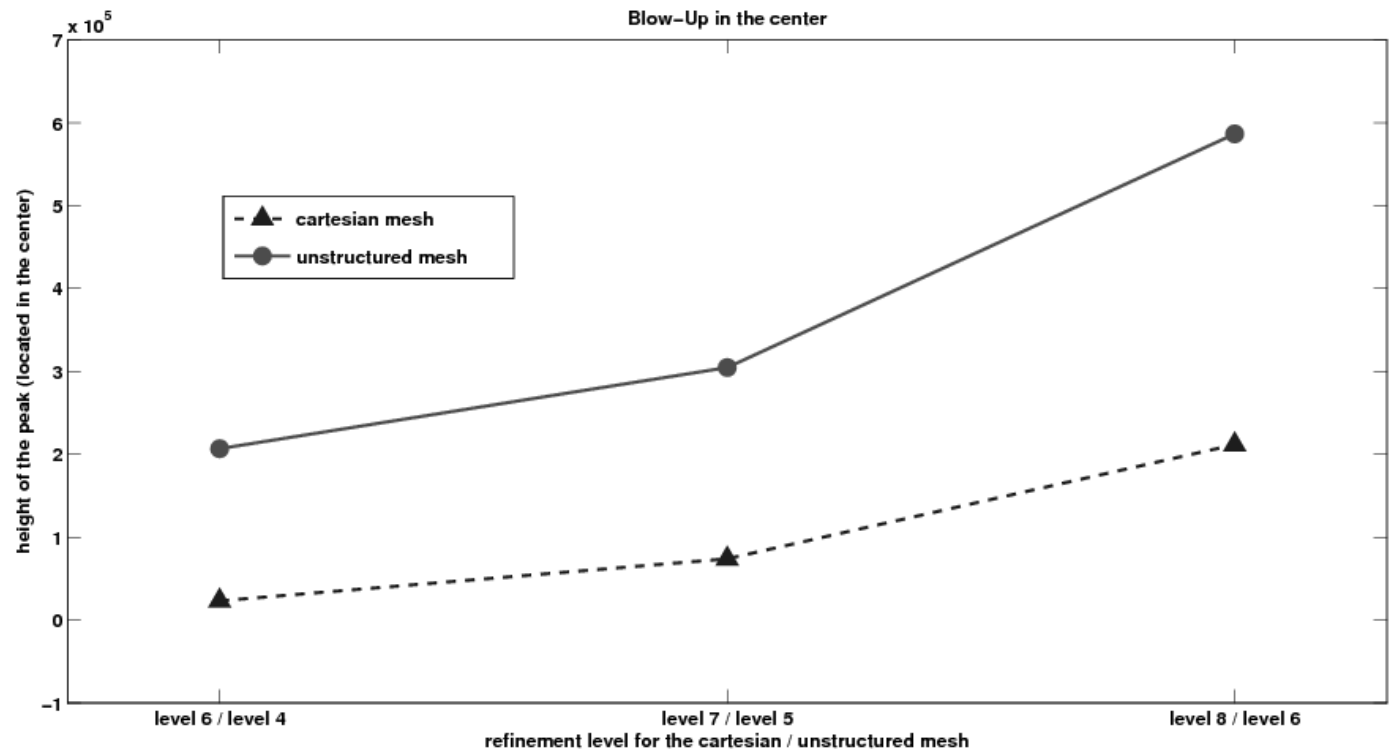

Fig. 3.6. Peak heights variation with mesh refinement. 


\subsection{Blow-up at the boundary of the domain}

In the second example, the system of chemotaxis equations (3.1)-(3.2) is solved subject to the initial conditions

$$
\begin{aligned}
& u_{0}(x, y)=1000 e^{-100\left((x-0.75)^{2}+(y-0.75)^{2}\right)} \\
& c_{0}(x, y)=0
\end{aligned}
$$

Since the initial chemoattractant concentration is zero, the blow-up is expected to occur much later than in the previous example. Therefore, simulations are performed with a larger time step $\Delta t=10^{-3}$. As time evolves, the solution of system (3.1)-(3.2) assumes a spiky form and moves towards the upper right corner of the domain. The results obtained with the standard Galerkin discretization are displayed in Fig. 3.7. Again, the cell density becomes negative, and nonphysical oscillations are observed in the corner. These problems can be cured using algebraic flux correction of FCT type, as demonstrated by the solutions in Fig. 3.8.
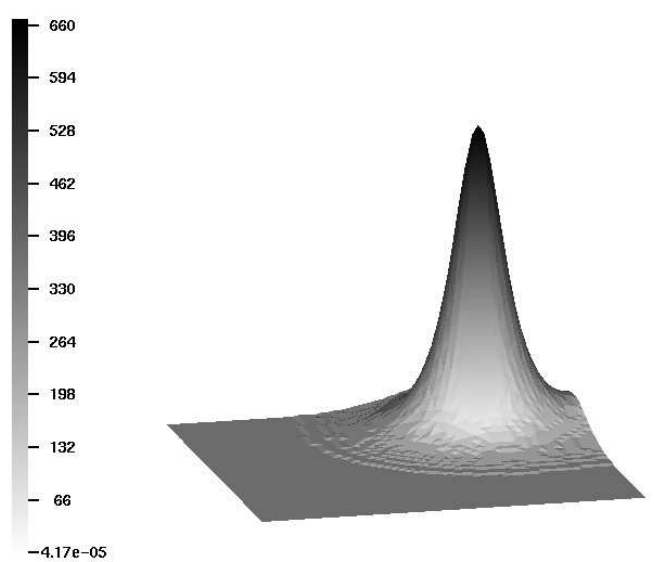

(a) $t=0.01$
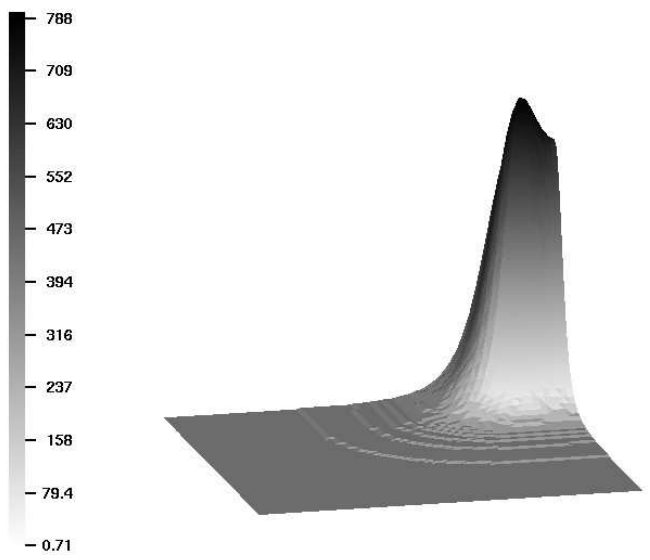

(c) $t=0.07$
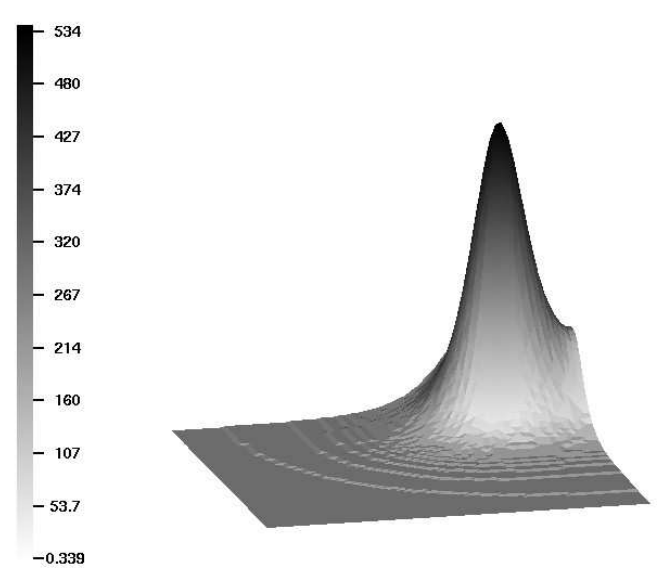

(b) $t=0.05$
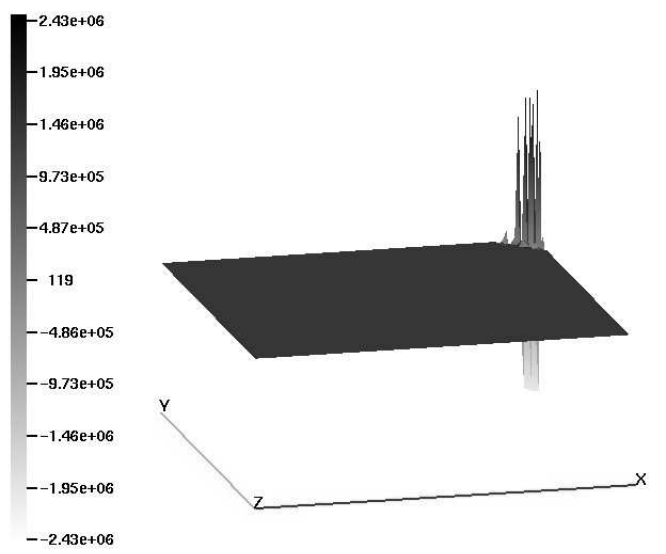

(d) $t=0.1$

Fig. 3.7. Blow-up in the corner, Galerkin scheme, $h=\frac{1}{128}, \Delta t=10^{-3}$. 

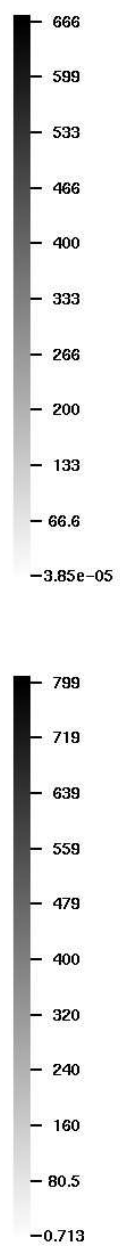

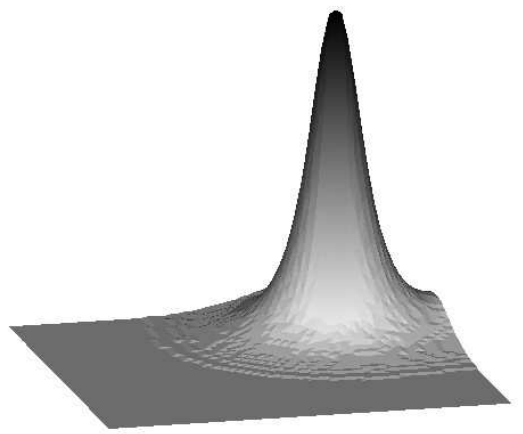

(a) $t=0.01$

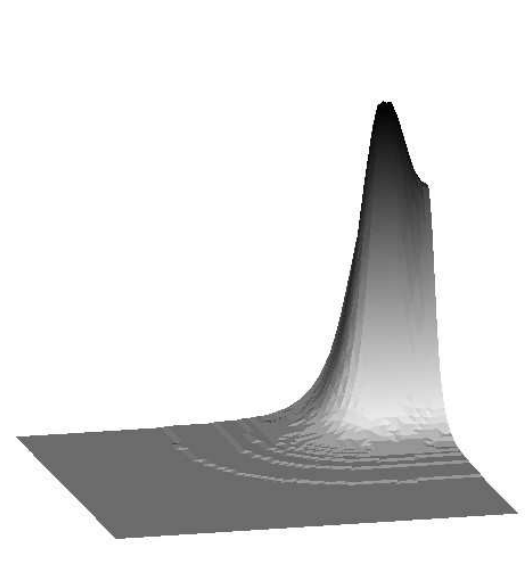

(c) $t=0.07$
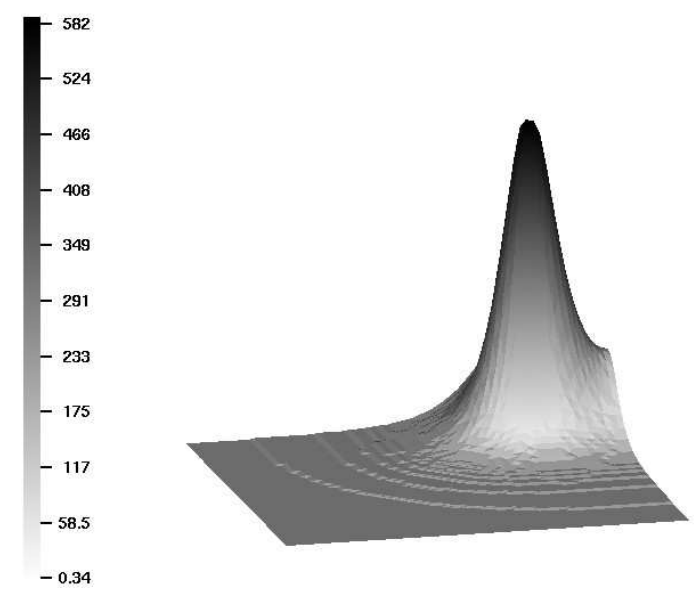

(b) $t=0.05$
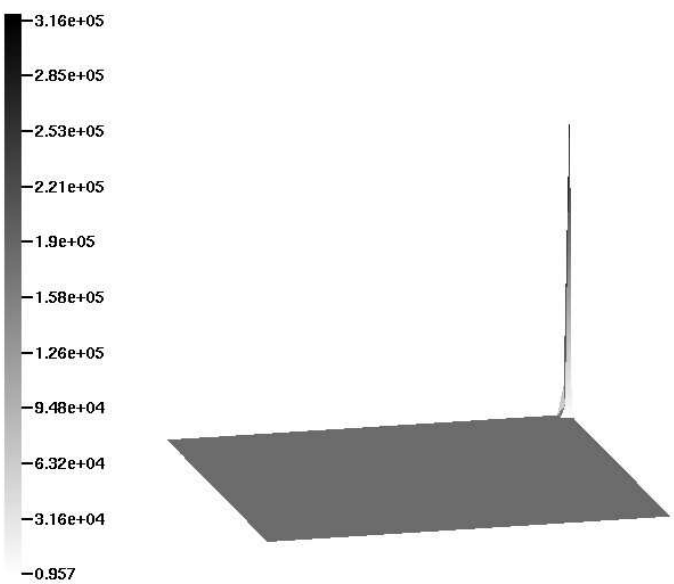

(d) $t=0.1$

Fig. 3.8. Blow-up in the corner, FEM-FCT scheme, $h=\frac{1}{128}, \Delta t=10^{-3}$.

The point of blow-up may depend on the geometry on the computational domain, as well as on the imposed boundary conditions [11]. For example, let $\Omega$ be a circle of radius 0.5 centered at the point $(0.5,0.5)$. A typical coarse mesh is depicted in Fig. 3.9 (a). The purpose of the numerical experiment to be performed is to find out if the blow-up point tends to any particular location. The peak of the initial profile $u_{0}$ is placed at the point $(0.6,0.6)$

$$
\begin{aligned}
& u_{0}(x, y)=1000 e^{-100\left((x-0.6)^{2}+(y-0.6)^{2}\right)}, \\
& c_{0}(x, y)=0 .
\end{aligned}
$$

All other settings are the same as in the case of the square domain. The FEM-FCT results in Fig. 3.9 (b,c,d) were obtained with 9216 bilinear elements. The distribution of the cell density moves in the radial direction and blows up at the boundary of the circle in finite time. 


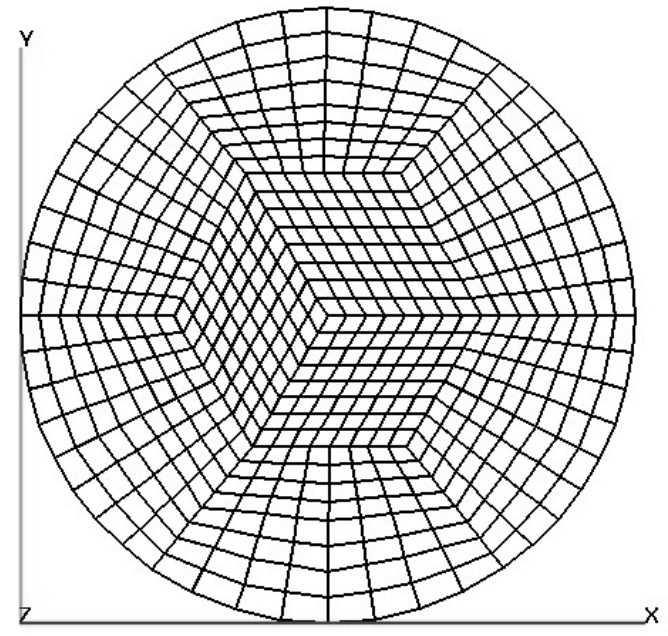

(a) coarse mesh
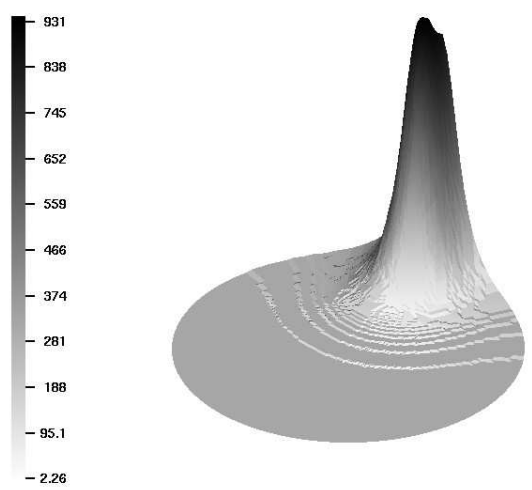

(c) $t=0.14$
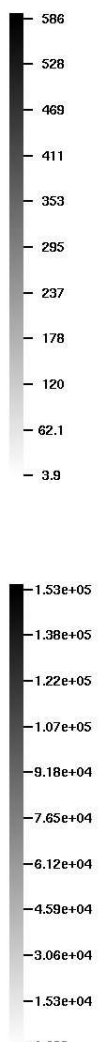

$-0.632$ (b) $t=0.085$
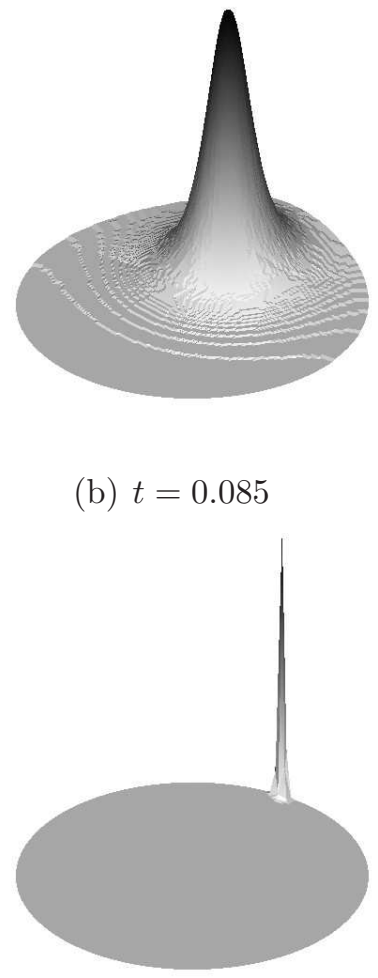

(d) $t=0.2$

Fig. 3.9. Blow-up at a circular boundary, FEM-FCT scheme, $\Delta t=10^{-3}$.

\subsection{Pattern formation}

In the last example, we consider a more complicated and realistic chemotaxis model. It describes the complex space-time patterns formed by motile cells of Escherichia coli. There are several different approaches to modeling the distribution of these bacteria. One of them leads to the following system of differential equations [5]:

$$
\begin{aligned}
u_{t} & =D_{1} \Delta u-\alpha \nabla \cdot\left(\frac{u}{(1+c)^{2}} \nabla c\right), \\
c_{t} & =D_{2} \Delta c+\beta \frac{w u^{2}}{\sigma+u^{2}} .
\end{aligned}
$$

For theoretical analysis, numerical algorithms, and simulation results we refer to [7, 16, 27].

In another model, proposed by Mimura and Tsujikawa [21], only the diffusion, the chemotaxis, and the growth of bacteria are taken into account. The corresponding PDE system reads

$$
\begin{aligned}
& u_{t}=D_{1} \Delta u-\chi \nabla \cdot(u \nabla c)+u^{2}(1-u), \\
& c_{t}=\Delta c-\beta c+u .
\end{aligned}
$$


For a detailed presentation of this approach see, e.g., [1, 2]. Although both systems (3.6)(3.7) and (3.8)-(3.9) model the space-time patterns formed by motile cells of Escherichia coli and fit the structure of (1.1)-(1.2), in this article we consider only the Mimura-Tsujikawa model (3.8)-(3.9) with $D_{1}=0.0625, \chi=8.5$, and $\beta=32$. These parameter settings are taken from $[1,2]$. The initial conditions are given by

$$
\begin{aligned}
& u_{0}(x, y)=1+\sigma(x, y), \\
& c_{0}(x, y)=1 / 32,
\end{aligned}
$$

where $\sigma(x, y)$ is a small perturbation defined as

$$
\sigma(x, y)= \begin{cases}\text { random, } & \text { if }\left\|\boldsymbol{x}-(8,8)^{T}\right\| \leqslant 1.5 \\ 0, & \text { otherwise. }\end{cases}
$$

Numerical simulations are performed in the square domain $\Omega=(0,16)^{2}$ discretized using a uniform mesh of conforming bilinear finite elements. The employed mesh size $h=1 / 8$ corresponds to 16384 cells. The time step is taken to be $\Delta t=0.1$. The solutions are very sensitive to the choice of parameters, especially $\chi$, $\sigma$, etc. Figure 3.10 illustrates the temporal evolution of the cell distribution obtained with the implicit FEM-FCT algorithm. The presented results are in quantitative agreement with those reported in $[1,2]$. The same formation patterns have been observed experimentally [5].

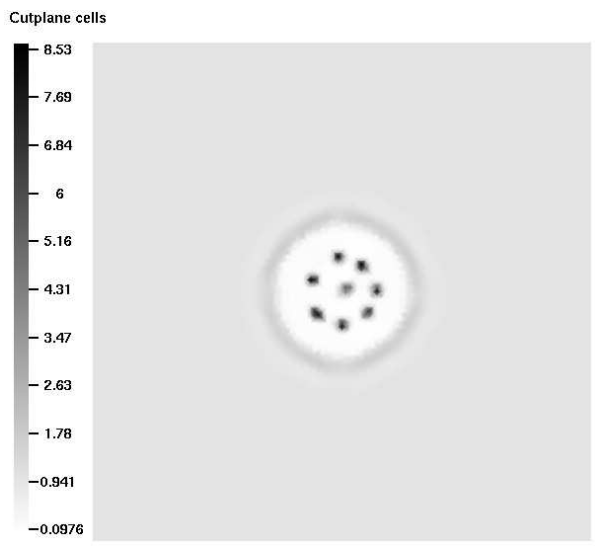

(a) $t=0.01$

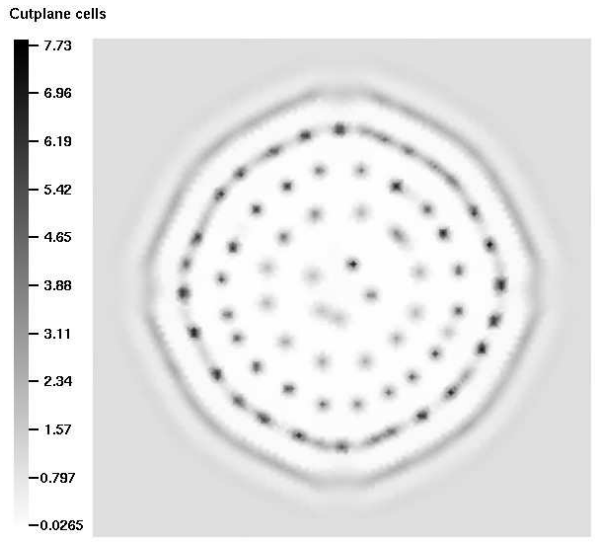

(c) $t=0.07$

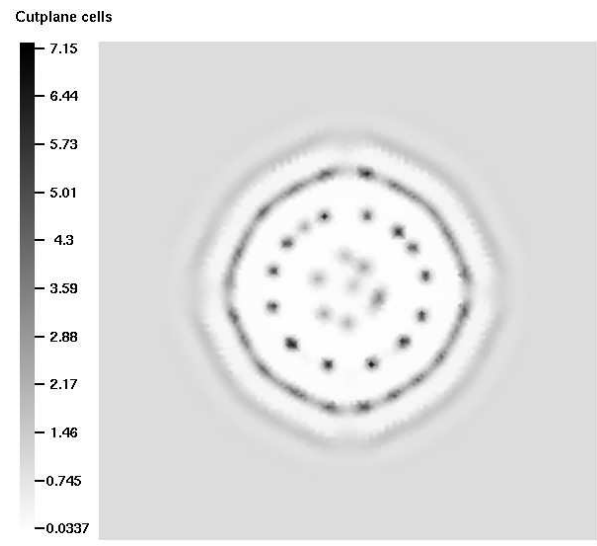

(b) $t=0.05$

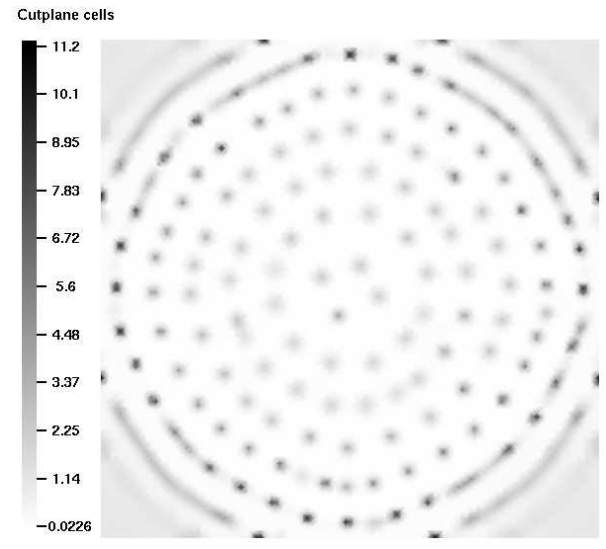

(d) $t=0.1$

Fig. 3.10. Pattern formation simulated with the FEM-FCT algorithm, $\Delta t=0.1, h=\frac{1}{8}$. 


\section{Conclusions}

An implicit flux-corrected transport algorithm has been developed for the unified form (1.1)(1.2) of chemotaxis models. Positivity constraints were enforced using a nonlinear blend of high- and low-order approximations. The employed limiting strategy is fully multidimensional and applicable to (multi-)linear finite element discretizations on unstructured meshes. The resultant scheme satisfies the discrete maximum principle and resolves steep gradients without excessive smearing. The local order of accuracy varies between first (low-order solution) and second (high-order solution), depending on the amount of artificial diffusion retained at the flux correction step. The robustness and efficiency of the linearized FEMFCT algorithm make it an attractive alternative to other stabilization techniques for the chemotaxis problems proposed in the literature [7, 9, 10, 16, 23, 28].

A preliminary numerical study of the implicit FEM-FCT scheme has been performed for the minimal Keller-Segel model. The flux-corrected Galerkin approximation has been shown to be sufficiently accurate and positivity-preserving, even in the case of solutions with sharp peaks that blow-up in the center or at the boundary of the domain. An example that illustrates the benefits of local mesh refinement was included. Furthermore, realistic simulation results were obtained for a representative model of chemotactical pattern formation. The proposed methodology is suitable for a 3D implementation and seems to be a promising approach to the numerical treatment of real-life chemotaxis problems in medicine and biology. Further research will concentrate on the design of FCT algorithms for (1.1)(1.2) with stronger coupling. The implications of the time-stepping method also call for a detailed investigation. Last but not least, a detailed quantitative comparison with existing numerical results $[7,9,10,25]$ is required to illustrate the pros and cons of different discretization/stabilization techniques.

Acknowledgments. This research was supported by the German Research Foundation, grant KU 1530/3.

\section{References}

1. M. Aida, T. Tsujikawa, M. Efendiev, A. Yagi, and M. Mimura, Lower estimate of the attractor dimension for a chemotaxis growth system, Journal of the London Mathematical Society, 74 (2006), no. 2, pp. $453-474$.

2. M. Aida and A. Yagi, Target pattern solutions for chemotaxis-growth system, Scientiae Mathematicae Japonicae, 59 (2004), no. 3, pp. 577-590.

3. A. Bonami, D. Hilhorst, E. Logak, and M. Mimura, Singular limit of chemotaxis-growth model, Adv. Differential Equations, 6 (2001), pp. 1173-1218.

4. J. P. Boris and D. L. Book, Flux-corrected transport. I. SHASTA, A fluid transport algorithm that works, J. Comput. Phys., 11 (1973), pp. 38-69.

5. E. O. Budrene and H. C. Berg, Dynamics of formation of symmetrical patterns by chemotactic bacteria, Nature, 376 (1995), no. 6535, pp. 49-53.

6. M. Burger, M. Di Francesco, and Y. Dolak-Stru, The Keller-Segel model for chemotaxis with prevention of overcrowding: Linear vs. nonlinear diffusion, SIAM J. Math. Anal., 38 (2006), pp. 1288-1315.

7. A. Chertock and A. Kurganov, A second-order positivity preserving central-upwind scheme for chemotaxis and haptotaxis models, Numer. Math., 111 (2008), pp. 169-205.

8. Y. Dolak and C. Schmeiser, The Keller-Segel model with logistic sensitivity function and small diffusivity, SIAM J. Appl. Math., 66 (2005), pp. 595-615.

9. Y. Epshteyn, Discontinuous Galerkin methods for the chemotaxis and haptotaxis models, J. Comput. Appl. Math., 224 (2009), no. 1, pp. 168-181.

10. Y. Epshteyn and A. Kurganov, New interior penalty discontinuous Galerkin methods for the KellerSegel chemotaxis model, SIAM J. Numer. Anal., 47 (2008), no. 1, pp. 386-408. 
11. F. Filbet, A finite volume scheme for the Patlak-Keller-Segel chemotaxis model, Numer. Math., 104 (2006), no. 4, pp. 457-488.

12. M. A. Herrero and J. J. L. Velazquez, A blow-up mechanism for a chemotaxis model, Ann. Sc. Norm. Super., 24 (1997), pp. 633-683.

13. T. Hillen and K. J. Painter, A user's guide to PDE models for chemotaxis, J. Math. Biol., 58 (2009), no. 1, pp. 183-217.

14. E. F. Keller and E. F. Segel, Initiation of slime mold aggregation viewed as an instability, J. Theor. Biol., 26 (1970), pp. 399-415.

15. E. F. Keller and E. F. Segel, Model for chemotaxis, J. Theor. Biol., 30 (1971), pp. 225-234.

16. B. S. Kirk and G. F. Carey, A parallel, adaptive finite element scheme for modeling chemotactic biological systems, Commun. Numer. Meth. Engrg.,(in press).

17. D. Kuzmin, Explicit and implicit FEM-FCT algorithms with flux linearization, J. Comput. Phys., 228 (2009), pp. 2517-2534.

18. D. Kuzmin and M. Möller, Algebraic flux correction I. Scalar conservation laws, in: D. KuZMIN, R. LÖHner, S. TureK (Eds.), Flux-Corrected Transport: Principles, Algorithms, and Applications, Springer, Berlin, (2005), pp. 155-206.

19. D. Kuzmin and S. Turek, Flux correction tools for finite elements, J. Comput. Phys., 175 (2002), pp. $525-558$.

20. I. R. Lapidus and R. Schiller, Model for the chemotactic response of a bacterial population, Biophys J., 16 (1976), no. 7, pp. 779-789.

21. M. Mimura and T. Tsujikawa, Aggregating pattern dynamics in a chemotaxis model including growth, Physica A, 230 (1996),pp. 499-543.

22. A. B. Potapov and T. Hillen, Metastability in chemotaxis models, J. Dyn. Diff. Eq., 17 (2005), pp. 293-330.

23. D. L. Ropp and J. N. Shadid, Stability of operator splitting methods for systems with indefinite operators: Advection-diffusion-reaction systems, J. Comput. Phys., (in press).

24. H. R. Schwetlick, Travelling fronts for multidimensional nonlinear transport equations, Analyse non linéaire, 17 (2000), no. 4, pp. 523-550.

25. N. Saito, Conservative upwind finite-element method for a simplified Keller-Segel system modelling chemotaxis, IMA J. Numer. Anal., 27 (2007), pp. 332-365.

26. T. Suzuki, Free energy and self-interacting particles, Boston: Birkhauser, 2005.

27. R. Tyson, S. R. Lubkin, and J. D. Murray, A minimal mechanism for bacteria pattern formation, Proc. Biol. Sci., 266 (1999), no. 1416, pp. 299-304.

28. R. Tyson, L. G. Stern, and R. J. LeVeque, Fractional step methods applied to a chemotaxis model, J. Math. Biol., 41 (1996), pp. 455-475.

29. S. T. Zalesak, Fully multidimensional flux-corrected transport algorithms for fluids, J. Comput. Phys., 31 (1979), pp. 335-362. 